\title{
Protection against $\alpha$-Naphthylisothiocyanate-Induced Acute Cholestasis in Mice by a Novel Taurine Conjugate of Ursodeoxycholate (UR-906)
}

\author{
Shigemasa Kinbara, Kaoru Ishizaki, Yoshishige Takeuchi, Norio Miyazawa and Tsutomu Araki \\ Pharmacological Research Laboratory, Research Laboratories, Tokyo Tanabe Co., Ltd., 33-3 Akabanekita 2-chome, Kita-ku, Tokyo 115, Japan \\ Received May 21, $1993 \quad$ Accepted July 19, 1993
}

\begin{abstract}
We investigated the effects of tauroursodeoxycholate (UR-906) and ursodeoxycholic acid (UDCA) on $\alpha$-naphthylisothiocyanate (ANIT)-induced cholestasis in mice. UR-906 and UDCA were given intravenously $2 \mathrm{hr}$ prior to and $2 \mathrm{hr}$ after ANIT $(80 \mathrm{mg} / \mathrm{kg}$, p.o.) treatment. The animals were sacrificed $48 \mathrm{hr}$ after ANIT administration, and serum markers of liver injury were examined. UR-906 prevented significant elevations in total bilirubin, bile acids and LDH. Furthermore, this drug reduced significant elevations in ALP and LAP. UDCA also prevented significant elevations in total bilirubin and LAP. These results indicate that UR-906 as well as UDCA has a beneficial effect against ANIT-induced cholestasis in mice.
\end{abstract}

Keywords: $\alpha$-Naphthylisothiocyanate (ANIT), Tauroursodeoxycholate (UR-906), Cholestasis

$\alpha$-Naphthylisothiocyanate (ANIT) induces hepatic lesions in experimental animals. The hepatic injuries are well-known to closely resemble biliary cirrhosis which occurs in humans $(1,2)$. Therefore, the animals treated with ANIT have been commonly used as an excellent model of intrahepatic cholestasis $(3,4)$. Ursodeoxycholic acid (UDCA) has previously been shown to improve liver function in patients with cholestatic disorders such as primary biliary cirrhosis (5) and primary sclerosing cholangitis. In experimental animals, UDCA is also known to have choleretic and cytoprotective actions. Several authors have suggested that the beneficial effect of UDCA may result from replacement of the more toxic endogenous bile acids with the less membrane-toxic UDCA. Tauroursodeoxycholate (UR-906) is a novel taurine conjugate of UDCA. Previous studies demonstrated that UR-906 prevented biliary protein excretion induced by taurochenodeoxycholic acid (TCDC), taurolithocholic acid (TLC)-induced cholestasis and liver injury in rats (6). Furthermore, Krol et al. (7) suggested that UR-906 reduced ductular proliferation and portal inflammation in bile-duct-ligated hamsters. These observations seem to suggest that UR-906 can prevent cholestasis and liver toxicity. Therefore, the present study was performed to further clarify the effect of UR-906 on ANIT-induced acute liver injury in mice.

Male ICR mice (Clea Japan, Inc., Tokyo) weighing $30-36 \mathrm{~g}$ were allowed food and water ad libitum through- out the experiments. Experimental cholestasis and hyperbilirunemia in animals were induced by oral administration of $80 \mathrm{mg} / \mathrm{kg}$ of ANIT (Tokyo Kasei Kogyo Co., Ltd., Tokyo) which was dissolved in olive oil. UR-906 (Tokyo Tanabe Co., Ltd., Tokyo) was dissolved in saline. UDCA (Tokyo Tanabe Co., Ltd.) was dissolved in $0.5 \mathrm{~N} \mathrm{NaOH}$ and adjusted to $\mathrm{pH} 8.5$ with $0.5 \mathrm{~N} \mathrm{HCl}$. Vehicle (saline), UR-906 and UDCA were given intravenously $2 \mathrm{hr}$ prior to and $2 \mathrm{hr}$ after ANIT treatment. Forty-eight hours after ANIT treatment, the mice were anesthetized with light ether, and blood samples were obtained by cardiac puncture. Serum total bilirubin, bile acids concentrations, LDH, LAP and ALP activities were all measured by commercial kits (Wako Pure Chemicals Co., Osaka). The control animals received injections of the corresponding vehicle, and the intact animals were non-treated mice. Each group contained 8 to 11 animals. Results were expressed as the mean \pm S.D. Statistical comparisons were made by Dunnett's multiple comparison test.

The biochemical findings are shown in Fig. 1. Vehicletreated mice showed significant increases in serum total bilirubin, bile acids, ALP, LAP and LDH at $48 \mathrm{hr}$ after ANIT administration. In contrast, administration of UR-906 significantly reduced all the ANIT-induced biochemical changes. Especially, UR-906 dose-dependently prevented a significant elevation in serum total bilirubin. Furthermore, this drug prevented significant increases in bile acids and LDH. UDCA at a dose of $300 \mu \mathrm{mol} / \mathrm{kg}$ 


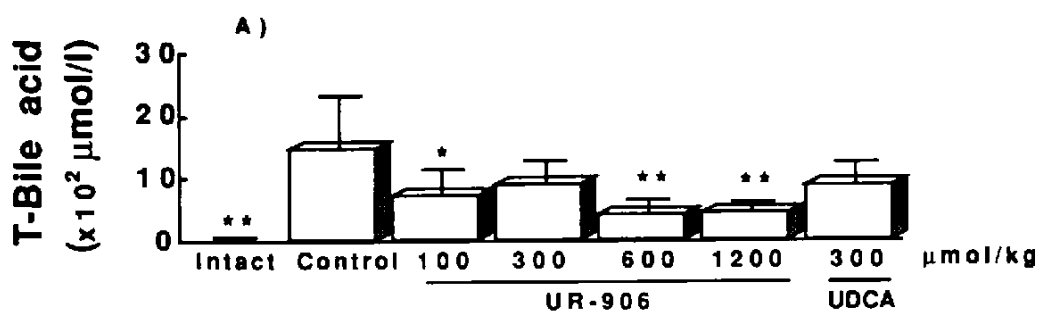

B)

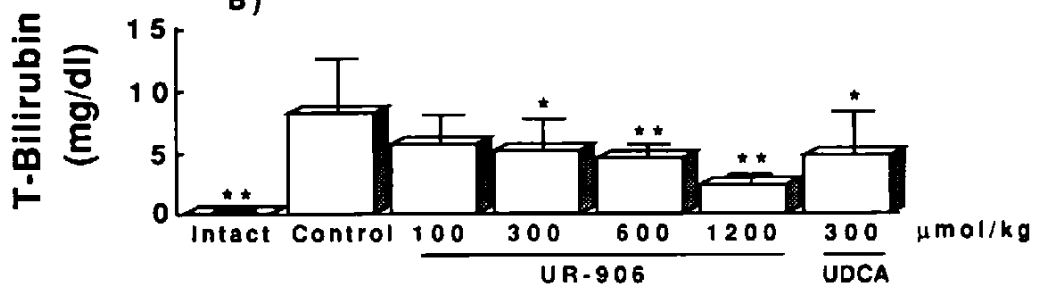

c)

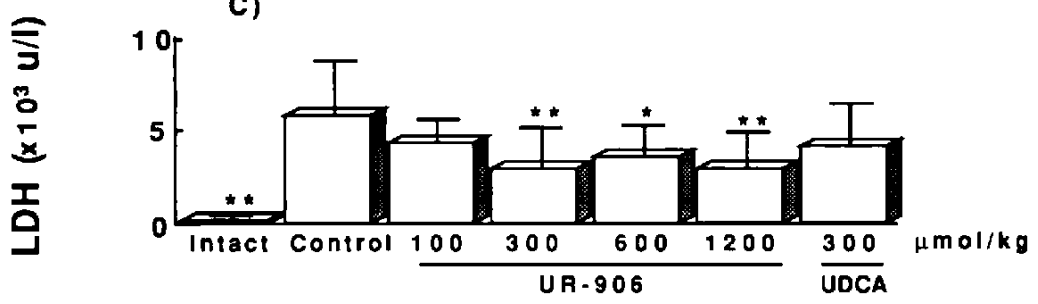

D)

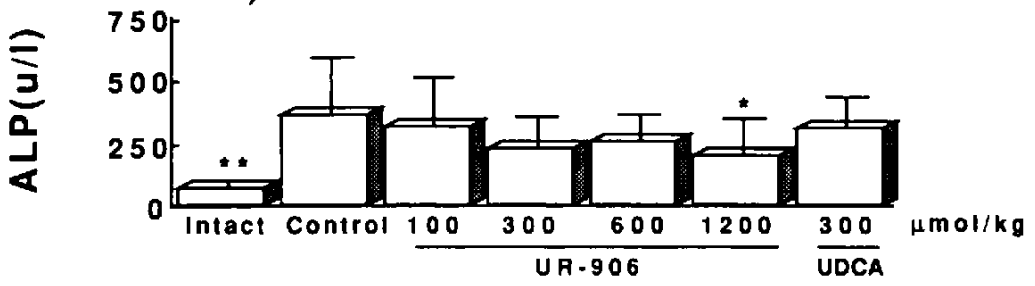

E)

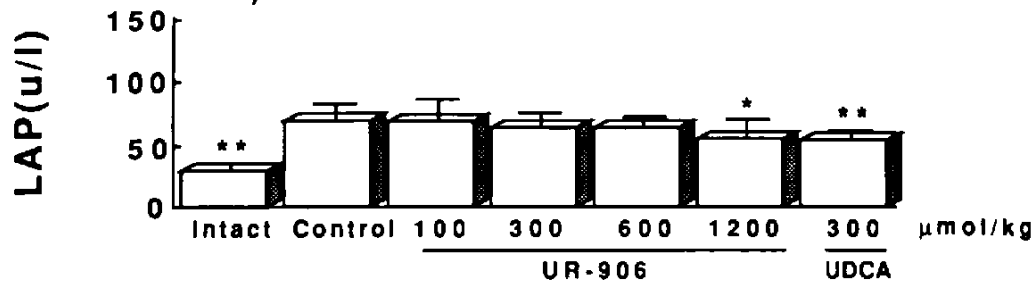

Fig. 1. Effect of UR-906 on biochemical changes in serum bile acids concentration (A), total bilirubin concentration (B), LDH activity (C), ALP activity (D) and LAP activity (E) at $48 \mathrm{hr}$ after ANIT treatment in mice. Values are expressed as the mean \pm S.D., $\mathbf{n}=8-11$. Significantly different from the control (vehicle-treated), ${ }^{*} \mathrm{P}<0.05,{ }^{* *} \mathrm{P}<0.01$ (Dunnett's multiple comparison test).

also reduced significant elevations in serum total bilirubin and LAP at $48 \mathrm{hr}$ after ANIT administration. However, this drug produced no significant changes in serum bile acids, ALP and LDH.

It has been well documented that a single dose of ANIT produces hepatic injuries in rats. The present study also indicated that ANIT administration in mice caused marked elevation in serum total bilirubin, bile acids, ALP, LAP and LDH. This phenomenon demonstrates that ANIT ad- ministration can produce transient cholestasis and liver injury in mice. ANIT is a cholestatic agent which causes damage to bile duct epithelial cells and hepatocytes, primarily in the periportal regions of liver $(8,9)$. The precise mechanisms for the toxicity of ANIT are presently unclear. However, much attention has focused on the role of hepatic, cytochrome P450-dependent, mixed-function oxidases (10). Therefore, ANIT may be bioactivated to a metabolite which is toxic specifically to bile duct epitheli- 
um. In the present study, UR-906 prevented significant elevations in serum total bilirubin, bile acids, ALP, LDH and LAP in ANIT-treated mice. This effect was especially noted in serum total bilirubin and bile acids concentrations. This finding seems to suggest that UR-906 is able to facilitate biliary excretion of bile acids and biliary or urinary excretion of bilirubin. Furthermore, UR-906 reduced significant elevations in ALP, LDH and LAP in ANIT-treated mice. The reason for this phenomenon is presently unknown, but it is conceivable that UR-906 attenuates the hepatotoxicity of ANIT via an increase of bile flow and bile salt excretion at the level of the liver or membrane stabilization. On the other hand, UDCA also prevented a significant elevation in serum total bilirubin and LAP in ANIT-treated mice. This beneficial effect of UDCA may also be produced by membrane stabilization or increase of bile flow and bile salt excretion at the level of the liver, as described previously (11). These results demonstrate that UR-906 as well as UDCA has a beneficial effect against ANIT-induced cholestasis in mice. They also suggest that UR-906 may be useful in the treatment of drug-induced cholestasis in humans. Further studies are needed to investigate the biochemical mechanisms for such effects.

\section{REFERENCES}

1 Popper, H., Rubin, E. and Schaffner, F.: The problem of primary biliary cirrhosis. Am. J. Med. 33, 807-810 (1962)

2 Boyer, J.L.: Definitive therapy for primary biliary cirrhosis:
Fact or fiction? Gastroenterology 95, 242-245 (1988)

3 Schaffner, F., Scharnbeck, H., Hutterer, F., Denk, H., Greim, H.A. and Popper, H.: Mechanism of cholestasis. VII. $\alpha-$ Naphthylisothiocyanate-induced jaundice. Lab. Invest. 28, $321-331$ (1973)

4 Fukumoto, Y.: Studies on alpha-naphthylisothiocyanate induced cholestasis. Acta Hepatol. Japon. 19, 647-656 (1978) (Abs. in English)

5 Matsuzaki, Y., Tanaka, N., Osuga, T., Aikawa, T., Shoda, J., Doi, M. and Nakano, M.: Improvement of biliary enzyme levels and itching as a result of long-term administration of ursodeoxycholic acid in primary biliary cirrhosis. Am. J. Gastroenterol. 85, 15-23 (1990)

6 Kitani, K., Ohta, M. and Kanai, S.: Tauroursodeoxycholate prevents biliary protein excretion induced by other bile salts in the rat. Am. J. Physiol, 248, G407-G417 (1985)

7 Krol, T., Kitamura, T., Miyai, K. and Hardison, W.: Tauroursodeoxycholate (TUDC) reduces ductular proliferation and portal inflammation in bile-duct-ligated hamsters. Hepatology 3, 881 (1983)

8 Ungar, H., Moran, E., Eisner, M. and Eliakim, M.: Rat intrahepatic biliary tract lesions from alpha-naphthylisothiocyanate. Arch. Pathol. 73, 427-435 (1962)

9 Goldfarb, S., Singer, E.J. and Popper, H.: Experimental cholangitis due to alpha-naphthylisothiocyanate (ANIT). Am. J. Pathol. 40, 685-698 (1962)

10 Fukumoto, Y., Okita, K., Oda, M., Ando, K., Matsuda, S., Watanabe, S., Kodama, T. and Takemoto, T.: Mechanism of alpha-naphthylisothiocyanate induced intrahepatic cholestasis: Studies of cytochrome P-450 in liver microsome and nucleus. Acta Hepatol. Japon. 22, 552-558 (1981) (Abs. in English)

11 Kitani, K. and Kanai, S.: Effect of ursodeoxycholate on the bile flow in the rat. Life Sci. 31, 1973-1985 (1982) 